\title{
Distributed Controller Design for Vehicle Platooning under Packet Drop Scenario
}

\author{
Kaushik Halder ${ }^{1}$, Umberto Montanaro ${ }^{1}$, Lee Gillam², Mehrdad Dianati ${ }^{3}$, David Oxtoby ${ }^{4}$, Alexandros \\ Mouzakitis ${ }^{4}$, Saber Fallah ${ }^{1}$ \\ 1. Connected Autonomous Vehicle Lab, Department of Mechanical Engineering Sciences, University of Surrey, \\ Guildford, GU2 7XH, UK (Email: \{k.halder, u.montanaro, s.fallah\}@surrey.ac.uk) \\ 2. Department of Computer Science, University of Surrey, Guildford, GU2 7XH, UK (Email: \\ l.gillam@surrey.ac.uk) \\ 3. WMG, International Manufacturing Centre, University of Warwick, Coventry, CV4 7AL UK (Email: \\ m.dianati@warwick.ac.uk). \\ 4. Jaguar Land Rover Limited, Coventry, CV3 4LF, UK (Email: \{doxtoby, amouzak1\}@jaguarlandrover.com)
}

\begin{abstract}
This paper proposes a distributed control strategy for homogeneous platoon systems with external disturbances under random packet drop scenario which can occur due to underlying network among the vehicles in a platoon. An linear matrix inequality (LMI) based approach is used to obtain the controller gains for ensuring the stability with bounded $H_{\infty}$ norm for such systems. Effectiveness of the proposed method is demonstrated with numerical results considering different network topologies in a platoon under single packet drop. The variation of $H_{\infty}$ norm bound for different number of platoon members under the different structure of network topologies and the packet drop has been studied in this paper.
\end{abstract}

Keywords—vehicle platoon, distributed controller, LMI, packet drop

\section{INTRODUCTION}

Vehicle platoon is a group of two or more connected autonomous vehicles (CAVs) where all vehicles travel with the same velocity while maintaining a small inter-vehicular distance within themselves. In a highway, the platoon of CAVs offers number of benefits like road safety, highway utility, and fuel economy etc. [1]. In a platoon, vehicles exchange information with their neighbours through wireless vehicle-to-vehicle (V2V) [2] and vehicle-to-infrastructure (V2I) [3] communication systems. These communication systems utilize the concepts of network topologies e.g. (i) predecessor following (PF), (ii) predecessor leader following (PLF), (iii) bidirectional PF (BPF) [4], (iv) bidirectional predecessor leader following (BPLF), (v) two PF (TPF) [5], (vi) two BPF (TBPF) [4] and (vii) all-to-all [6] etc. relating the way of information exchange between vehicles in a platoon. However, the reliability of wireless communication network depends largely on bandwidth allocation, external disturbances, signal strength etc., which ultimately leads to packet drop and/or delay in data transmission. This packet drops and/or delay largely affects the stability and performance of the vehicle platoon system [7] due to presence of wireless communication network in it. Therefore, it is quite challenging to ensure the stability and maintain the desired performances (maintaining the same velocity and inter-vehicular distance) by designing the appropriate controller for the vehicle platoon system under different network topologies with packet drop.

The importance of network topology has been described for designing the controller to analyse the closed-loop stability and co-operative motion of the vehicles in a platoon in [5]. Recently, number of advanced control strategies have been implemented to achieve better performances of vehicle platoon using different network topologies [3, 8-11]. In [8], a distributed receding horizon controller has been designed for platoons under the PF topology while [9] extended the results of [8] with unidirectional topology. Using graph theory and Routh-Hurwitz criteria, the stabilizing threshold of controller gains has been derived in [2]. A distributed $H_{\infty}$ controller has been designed for homogeneous platoon with undirected topology using LMI approach in [10]. An adaptive control strategy has been adopted for adjusting the gains of the distributed controller as a function of mismatched states of the platoon members in [6]. However, these abovementioned methods do not ensure stability and performances under network imperfections (e.g. packet drop and/or delay), parametric uncertainty such as mass of passenger vehicles or engine time constant [12] and external disturbances such as lead vehicle's acceleration, wind gust or road slope [10, 1213 ] acting on the vehicles in a platoon.

Although packet drop, communication delays, parametric uncertainty and external disturbances all have been investigated by researchers separately $[7,10]$ or in some combinations [13] for the vehicle platoon control problem but combination of all these issues remains a challenging research problem $[9,10]$. For example, for short range wireless communication environment among the vehicles in a platoon, a decentralized model predictive controller has been designed under low and high communication latency for longitudinal platoon control problem in [14]. Tang et al. [7] have utilized the concept of network topology for communication systems [5] between vehicles in a platoon and have designed the LMI based distributed controller to achieve the co-ordinated motion of 
each vehicle under packet loss. Other approaches have used co-operative adaptive cruise control to analyse string stability and performance of platoons under packet drop $[15,16]$ and communication delay $[17,18]$ when vehicles are wirelessly connected. However, to the best our knowledge, very few researchers have proposed control strategies with networked packet drop or delay for vehicle platoon control under parametric uncertainty and/or external disturbances $[13,19]$. An LMI based distributed $H_{\infty}$ controller has been designed for heterogeneous vehicle platoon control under the parametric uncertainty, external disturbances and communication delays in [19]. However, the method proposed in [19] only considered PLF network topology for the communication among the vehicles in a platoon. As an extension of the work [19], our method proposes a distributed controller design methodology for vehicle platoon under random single packet drop and external disturbances considering the network topology for communication between vehicles are generic (i.e. valid for different network topologies (i)-(vii)) as reported in [4, 5].

This paper proposes a robust distributed control design methodology for homogeneous vehicle platoon under random packet drop. As in [10], the longitudinal dynamics of homogeneous platoon with disturbances has been considered in this work. However, to design the distributed controller for the homogeneous platoon with external disturbances, first, we recast the platoon control problem as a synchronization problem of a multi agent system $[8,11]$, i.e. leader-following consensus under single packet drop scenario using Bernoulli modelling of packet drop distribution is used. Next, we have used Lyapunov based LMI approach to obtain the controller gains with the satisfaction of certain bounded $H_{\infty}$ norm which ensure the stability and maintain desired intervehicular distance for such systems. The effect of various network topologies with random packet drop rate on $H_{\infty}$ norm bound for different numbers of platoon members has been studied in this paper. In addition, for different platoon members, the effect of leader vehicle's information to the follower vehicles in a platoon under network topologies and packet drop has also been studied by analysing the robust performance measures i.e. $H_{\infty}$ norm bound $\gamma$.

The rest of the paper is organized as follows. Section II describes platoon modelling and control objectives of platoon for synchronization problem under single packet drop scenario. Then to achieve the control objectives for a platoon, the LMI based distributed controller design approach has been described in Section III. Section IV represents the detail derivation of proposed methodology. Numerical results have been shown in section $\mathrm{V}$ to analyse the effectiveness of proposed method. The final conclusions have been presented in Section VI followed by references.

\section{Platoon Modeling AND CONTROL OBJectVes}

In this paper, the platoon problem can be considered as a synchronization problem of a networked dynamical systems with a pinner node $[5,11]$ where a set of agents (i.e. nodes) interact among themselves through network and these nodes are controlled in such a way that the dynamics of all nodes converge towards the pinner node i.e. leader of a platoon. The main components of a network of dynamical systems are (i) individual node dynamics which describes the evaluation of each node when not coupled with the network; (ii) different network topology which describe the interaction among nodes; (iii) control action to each node for steering the node dynamics to the pinner's trajectory. This section describes the modelling of the platoon network topology and longitudinal dynamics of the vehicles in a platoon which represent the node. The control objectives of platoon are also discussed in this section. Since this paper deals with a synchronization problem [6] under communication topology, the follower vehicles may be connected with a pinner node i.e. leader and it is further assumed that all the followers may receive information from the leader, but the followers will not send any information to the leader.

\section{A. Modelling of the Platoon Network Topology and Packet drop}

The communication topology of the $N$ followers (nodes) can be represented by the graph $G_{N}=\left(V_{N}, E_{N}\right)$, where $V_{N}=\{1,2, \ldots, N\}$ defines a set of vertices or nodes and $E \subseteq V_{N} \times V_{N}$ defines a set of arcs or edges. The pair $(j, i) \in E_{N}$ indicates the $i^{\text {th }}$ vehicle receives the information from $j^{\text {th }}$ vehicle. An adjacency matrix $A_{N}=\left[a_{i j}\right] \in \mathbb{R}^{N \times N}$ can be defined based on the edges $E_{N}$. The generic entry $a_{i j}$ of the adjacency matrix defines $a_{i j}=1$ when $(j, i) \in E_{N}$ and $a_{i j}=0$ otherwise i.e. $a_{i j}=1$ implies when the $i^{\text {th }}$ vehicle receives the information from $j^{\text {th }}$ vehicle while $a_{i i}=0$ represents no self-loop in the network. The degree $D_{N}$ of the graph is represented by the number of edges pointing to the follower. For such case the Laplacian matrix $L=\left[l_{i j}\right] \in \mathbb{R}^{N \times N}$ which can be represented by $L=D_{N}-A_{N}$ where $l_{i i}=\sum_{j \neq i} a_{i j}$ and $l_{i j}=-a_{i j} \forall i \neq j$. If the network topology considers the leader (i.e. leader of the platoon or pinner of the network) then the graph $G_{N}$ is augmented with node 0 and the modified graph is $G_{N+1}=\left(V_{N+1}, E_{N+1}\right)$ where $V_{N+1}=\{0,1, \ldots, N\}$ and $E_{N+1} \subseteq V_{N+1} \times V_{N+1}$, also representing the pinner of the network. The corresponding adjacency matrix is $A_{N+1}=\left[a_{i j}\right] \in \mathbb{R}^{(N+1) \times(N+1)} \quad$ with $a_{0 j}=0, j=0,1, \ldots, N$ indicates that the followers are not sending information to the leader, $a_{i 0}=1, i=1,2, \ldots, N$ indicates that the followers are receiving information from the leader and finally $a_{i 0}=0$ indicates otherwise. $N_{i}$ denotes the neighbour set in $G_{N+1}$ for the node $i, \quad i=1,2, \ldots, \mathrm{N}$ i.e. $N_{i}=\left\{j \in\{0,1, \ldots, N\}: a_{i j}=1\right\}$.

Let us consider the stochastic variable $\theta_{j i}(k) \in\{0,1\}$ is satisfying Bernoulli distribution of packet drop in the communication link among the vehicles. $\theta_{j i}(k)=0$ represents the follower $j$ will receive the information or packet from $i^{\text {th }}$ follower and $\theta_{j i}(k)=1$ represents packet or information is lost. Under such scenario the following holds: $\operatorname{Prob}\left(\theta_{i j}(k)=1\right)=E\left(\theta_{i j}(k)\right)=r$ 
and

$E\left(1-\theta_{i j}(k)\right)=1-r$,

where $r$ is the packet drop rate in the communication link among the vehicles.

\section{B. Modelling of the Longitudinal Vehicle Dynamics}

In a vehicle platoon, the nonlinear longitudinal dynamics of the vehicles can be represented by a third order linearized differential equation due to its satisfactory trade-off between accuracy and simplicity $[5,10]$. The third order model of $i^{\text {th }}$ vehicle with disturbance input in homogeneous platoon considering the states $\mathbf{x}_{i}^{T}(t)=\left[\begin{array}{lll}s_{i} & v_{i} & a_{i}\end{array}\right]$ is represented in state space form as:

$$
\begin{aligned}
\dot{\mathbf{x}}_{i}(t) & =\mathbf{A} \mathbf{x}_{i}(t)+\mathbf{B} u_{i}(t)+\mathbf{B} w_{i}(t) \\
y_{i}(t) & =\mathbf{C} \mathbf{x}_{i}(t)
\end{aligned}
$$

where $u_{i}(t) \in \mathbb{R}^{m}, w_{i}(t) \in \mathbb{R}^{m} \in L_{2}[0, \infty)$ and $y_{i}(t) \in \mathbb{R}^{q}$ represents system input, exogenous disturbance input and output matrices respectively with appropriate dimension and

$$
\mathbf{A}=\left[\begin{array}{ccc}
0 & 1 & 0 \\
0 & 0 & 1 \\
0 & 0 & -1 / \tau
\end{array}\right], \quad \mathbf{B}=\left[\begin{array}{c}
0 \\
0 \\
1 / \tau
\end{array}\right], \quad \mathbf{C}=\left[\begin{array}{lll}
1 & 0 & 0
\end{array}\right] .
$$

In (4), $s_{i}, v_{i}, a_{i}, \tau$ represents the position, velocity, acceleration and time lag respectively and $i=1,2, \ldots, N$ represents the $i^{\text {th }}$ follower vehicles.

The continuous time system (3) can be discretized using specified sampling time $T_{s}$ and the corresponding equivalent zero order hold $(\mathrm{ZOH})$ discretized system is

$$
\begin{aligned}
& \mathbf{x}_{i}(k+1)=\mathbf{A}_{d} \mathbf{x}_{i}(k)+\mathbf{B}_{d} u_{i}(k)+\mathbf{B}_{d} w_{i}(k) \\
& y_{i}(k)=\mathbf{C} \mathbf{x}_{i}(k)
\end{aligned}
$$

where $\mathbf{A}_{d}=e^{\mathbf{A} T_{s}}$ and $\mathbf{B}_{d}=\int_{0}^{T_{s}} e^{\mathbf{A} \tau} \mathbf{B} d \tau$.

In discrete time, the dynamics of leader vehicle $(i=0)$ with constant velocity can be represented as:

$$
\begin{aligned}
& \mathbf{x}_{0}(k+1)=\mathbf{A}_{d} \mathbf{x}_{0}(k) \\
& y_{0}(k)=\mathbf{C} \mathbf{x}_{0}(k)
\end{aligned}
$$

\section{Platoon Control Objectives}

The control objective of the platoon is categorized in two ways [6]: (i) impose leader velocity to all followers; (ii) maintain specified inter-vehicular distance between two consecutive vehicles in a platoon. These control objectives can be described by the following definition:

Definition 1 [20]: platoon of vehicles represented by leaderfollowing system is said to be consensus if it is satisfying the following under any initial conditions:

$$
\lim _{k \rightarrow \infty}\left\|\mathbf{x}_{i}(k)-\mathbf{x}_{0}(k)\right\|=\lim _{k \rightarrow \infty}\left\|\mathbf{e}_{i}(k)\right\|=0 \quad \forall i=1,2, \ldots, N,(7)
$$

where $\mathbf{e}_{i}(k)$ represents the tracking error.

Now, to achieve the above-mentioned control objective of a platoon, the controller is assumed to be static and identical for all the followers and then the corresponding distributed control law can be represented as:

$$
u_{i}(k)=\mathbf{K} \sum_{\substack{j=0 \\ j \neq i}}^{N} a_{i j}\left[\mathbf{x}_{i}(k)-\mathbf{x}_{j}(k)\right], \quad i=1,2, \ldots, N
$$

where, $\mathbf{K}=\left[\begin{array}{lll}-K_{s} & -K_{v} & -K_{a}\end{array}\right]$ represents the identical controller gain, $a_{i j} \in\{0,1\}$ is the $(i, j)$ entry of adjacency matrix $A_{N+1}=\left[a_{i j}\right] \in \mathbb{R}^{(N+1) \times(N+1)}$.

\section{STABILITY ANALYSIS AND CONTROLLER DESIGN UNDER PACKET DROP}

This section describes the packet drop modelling in the communication link of a vehicle platoon and then LMI based approach is used to analyse the stability and design distributed identical controller with bounded $H_{\infty}$ norm for such systems under the packet drop scenario.

\section{A. Modelling of the Packet drop for Vehicle Platoon}

Network packet drop modelled as a Bernoulli process can be used for the transmitted state vector $\mathbf{x}_{j}(k)$ as:

$$
\overline{\mathbf{x}}_{j}(k)=\left(1-\theta_{j i}(k)\right) \mathbf{x}_{j}(k)+\theta_{j i}(k) \mathbf{x}_{j}(k-1)
$$

Correspondingly,

$$
\overline{\mathbf{x}}_{i}(k)=\left(1-\theta_{i j}(k)\right) \mathbf{x}_{i}(k)+\theta_{i j}(k) \mathbf{x}_{i}(k-1)
$$

since follower $i$ knows whether it has received the information in the form of packet from $j^{\text {th }}$ follower or not at $k T_{s}$ instant. Here, we assume $\theta_{j i}(k)=\theta_{i j}(k)$ which defines that a specific link failure affects the packet drop in the both direction and all the link failure is asynchronous with same failure rate.

\section{B. Stability Analysis and Controller Design}

This sub-section describes the stability analysis and identical controller design with bounded $H_{\infty}$ norm for vehicles of platoon under the packet drop scenario. Considering the packet drop in the transmitted state vectors among the vehicles in a platoon and using (9), (10) and $\theta_{j i}(k)=\theta_{i j}(k)$ in (8), the distributed control law yields:

$$
u_{i}(k)=\mathbf{K} \sum_{\substack{j=0 \\
j \neq i}}^{N} a_{i j}\left[\begin{array}{l}
\left(1-\theta_{i j}(k)\right) \mathbf{x}_{i}(k)+\theta_{i j}(k) \mathbf{x}_{i}(k-1) \\
-\left(1-\theta_{i j}(k)\right) \mathbf{x}_{j}(k)-\theta_{i j}(k) \mathbf{x}_{j}(k-1)
\end{array}\right]
$$

$i=1,2, \ldots, N$.

Aim of this paper is to design the distributed control law (8) for vehicle platoon systems under random packet drop by ensuring the mean square stability and bounded $H_{\infty}$ norm $\gamma$ to reach the consensus of the system (17).

Definition 2 [11]: The system (17) is said to be mean square stable (MSS) if $\lim _{k \rightarrow \infty} E\left(\|\mathbf{e}(k)\|^{2}\right)=0$ for any initial state $\mathbf{e}(0) \in \mathbb{R}^{n}[17]$.

Definition 3 [21]: The output error dynamics $\left(\mathbf{y}_{e}\right)$ are said to have a bounded $H_{\infty}$ norm $\gamma>0$, when the closed-loop system is MSS and

$$
\sum_{k=0}^{\infty} E\left(\left\|\mathbf{y}_{e}(k)\right\|^{2}\right) \leq \gamma^{2} \sum_{k=0}^{\infty} E\left(\|\mathbf{w}(k)\|^{2}\right) \forall \mathbf{w}(k) \neq 0 \text {, and } \mathbf{e}(0)=0
$$

Now, this paper addresses the distributed controller design methodology satisfying MSS with bounded $H_{\infty}$ norm $\gamma$ for 
the closed loop system (17) using LMI approach which is stated as Theorem 1, below.

Theorem 1 provides sufficient conditions for the control gain $\mathbf{K}$ in (8) to guarantee that the closed loop system is MSS with a given $H_{\infty}$ norm bound $\gamma$.

Theorem 1: Given closed loop system (17) with packet loss modelled as in (1)-(2) with mean packet loss rate $r$ and the control action, then closed-loop system is MSS with bounded $H_{\infty}$ norm $\gamma$ if there exists

$\mathbf{P}=\mathbf{P}^{T}=\mathbf{I}_{N} \otimes \mathbf{P}_{0}>\mathbf{0}, \mathbf{Q}=\mathbf{Q}^{T}=\mathbf{I}_{N} \otimes \mathbf{Q}_{0}>\mathbf{0} \in \mathbb{R}^{n N \times n N} \quad$ such that

$$
\left[\begin{array}{ccc}
-\mathbf{P}^{-1} & * & * \\
\mathbf{0} & -\mathbf{P}^{-1} \mathbf{Q} \mathbf{P}^{-1} & * \\
\mathbf{0} & \mathbf{0} & -\gamma^{2} \otimes \mathbf{I}_{N} \\
\left(\left(\mathbf{I}_{N} \otimes \mathbf{A}_{d}\right) \mathbf{P}^{-1}+\tilde{\mathbf{L}}(1-r) \otimes \mathbf{B}_{d} \mathbf{Y}\right) & \tilde{\mathbf{L}} r \otimes \mathbf{B}_{d} \mathbf{Y} & \mathbf{I}_{N} \otimes \mathbf{B}_{d} \\
\mathbf{P}^{-1} & \mathbf{0} & \mathbf{0} \\
\left(\mathbf{I}_{N} \otimes \mathbf{C}\right) \mathbf{P}^{-1} & \mathbf{0} & \mathbf{0}
\end{array}\right.
$$

where, $\mathbf{P}^{-1}=\overline{\mathbf{P}}, \mathbf{Q}^{-1}=\overline{\mathbf{Q}}$ and the control gain in (8) is selected as $\mathbf{K}=\mathbf{Y} \mathbf{P}_{0}=\mathbf{Y} \overline{\mathbf{P}}_{0}^{-1} \in \mathbb{R}^{1 \times n}$.

\section{MAIN RESULTS AND PROOF}

This section uses the error dynamic model of the vehicle platoon to establish the stability criteria and design the distributed identical controller based on Lyapunov theory. Hence, first we derive the model of closed loop error dynamics subsequently using Lyapunov-like function, Theorem 1 presented in section III is proved for the platoon of vehicles.

\section{A. Modelling of Error Dynamics}

The followers (5) will be following the leader's dynamics (6) when the control law (11) is applied. Under this scenario, the error dynamics for the $i^{\text {th }}$ follower can be represented as:

$$
\begin{aligned}
& \mathbf{e}_{i}(k+1)=\mathbf{x}_{i}(k+1)-\mathbf{x}_{0}(k+1) \\
& =\mathbf{A}_{d} \mathbf{x}_{i}(k)+\mathbf{B}_{d} w_{i}(k)-\mathbf{A}_{d} \mathbf{x}_{0}(k) \\
& +\mathbf{B}_{d} \mathbf{K} \sum_{\substack{j=0 \\
j \neq i}}^{N} a_{i j}\left[\begin{array}{l}
\left(1-\theta_{i j}(k)\right) \mathbf{x}_{i}(k)+\theta_{i j}(k) \mathbf{x}_{i}(k-1) \\
-\left(1-\theta_{i j}(k)\right) \mathbf{x}_{j}(k)-\theta_{i j}(k) \mathbf{x}_{j}(k-1)
\end{array}\right]
\end{aligned}
$$

$$
\begin{aligned}
& =\mathbf{A}_{d}\left(\mathbf{x}_{i}(k)-\mathbf{x}_{0}(k)\right)+\mathbf{B}_{d} w_{i}(k) \\
& +\mathbf{B}_{d} \mathbf{K} \sum_{\substack{j=0 \\
j \neq i}}^{N} a_{i j}\left[\begin{array}{l}
\left(1-\theta_{i j}(k)\right)\left(\mathbf{x}_{i}(k)-\mathbf{x}_{0}(k)-\mathbf{x}_{j}(k)+\mathbf{x}_{0}(k)\right)- \\
\theta_{i j}(k)\left(\mathbf{x}_{i}(k-1)-\mathbf{x}_{0}(k-1)-\mathbf{x}_{j}(k-1)+\mathbf{x}_{0}(k-1)\right)
\end{array}\right]
\end{aligned}
$$

The error dynamics (15) for $i^{\text {th }}$ the follower can be re-written using (7) as:

$$
\begin{aligned}
& \mathbf{e}_{i}(k+1)=\mathbf{A}_{d} \mathbf{e}_{i}(k)+\mathbf{B}_{d} w_{i}(k) \\
&+\mathbf{B}_{d} \mathbf{K} \sum_{\substack{j=0 \\
j \neq i}}^{N} a_{i j}\left[\begin{array}{l}
\left(1-\theta_{i j}(k)\right)\left(\mathbf{e}_{i}(k)-\mathbf{e}_{j}(k)\right) \\
-\theta_{i j}(k)\left(\mathbf{e}_{i}(k-1)-\mathbf{e}_{j}(k-1)\right)
\end{array}\right] \\
& y_{e_{i}}(k)=y_{i}(k)-y_{0}(k)=\mathbf{C}\left(\mathbf{x}_{i}(k)-\mathbf{x}_{0}(k)\right)=\mathbf{C e}_{i}(k) .
\end{aligned}
$$

Therefore, the error dynamics of all the followers in closed loop can be represented with the augmented states

$$
\begin{aligned}
& \mathbf{e}(k)=\left[\mathbf{e}_{1}^{T}(k), \mathbf{e}_{2}^{T}(k), \ldots, \mathbf{e}_{N}^{T}(k)\right]^{T} \\
& \mathbf{w}(k)=\left[w_{1}^{T}(k), w_{2}^{T}(k), \ldots, w_{N}^{T}(k)\right]^{T} \text { as: } \\
& \mathbf{e}(k+1)=\left(\mathbf{I}_{N} \otimes \mathbf{A}_{d}\right) \mathbf{e}(k)+\left(\mathbf{L}_{1} \otimes \mathbf{B}_{d} \mathbf{K}\right) \mathbf{e}(k) \\
& +\left(\mathbf{L}_{2} \otimes \mathbf{B}_{d} \mathbf{K}\right) \mathbf{e}(k-1)+\left(\mathbf{I}_{N} \otimes \mathbf{B}_{d}\right) \mathbf{w}(k), \\
& \mathbf{y}_{e}(k)=\left(\mathbf{I}_{N} \otimes \mathbf{C}\right) \mathbf{e}(k)
\end{aligned}
$$

where,

$\mathbf{L}_{1}=\left[\begin{array}{cccc}\sum_{j \in N_{i}} a_{1 j}\left(1-\theta_{1 j}(k)\right) & -a_{12}\left(1-\theta_{12}(k)\right) & \ldots & -a_{1 N}\left(1-\theta_{1 N}(k)\right) \\ -a_{21}\left(1-\theta_{21}(k)\right) & \sum_{j \in N_{i}} a_{2 j}\left(1-\theta_{2 j}(k)\right) & \ldots & -a_{2 N}\left(1-\theta_{2 N}(k)\right) \\ \vdots & \ddots & \ddots & \vdots \\ -a_{N 1}\left(1-\theta_{N 1}(k)\right) & -a_{N 2}\left(1-\theta_{N 2}(k)\right) & \ldots & \sum_{j \in N_{i}} a_{N j}\left(1-\theta_{N j}(k)\right)\end{array}\right]$

$$
\mathbf{L}_{2}=\left[\begin{array}{cccc}
\sum_{j \in N_{i}} a_{1 j} \theta_{1 j}(k) & -a_{12} \theta_{12}(k) & \ldots & -a_{1 N} \theta_{1 N}(k) \\
-a_{21} \theta_{21}(k) & \sum_{j \in N_{i}} a_{2 j} \theta_{2 j}(k) & \ldots & -a_{2 N} \theta_{2 N}(k) \\
\vdots & \ddots & \ddots & \vdots \\
-a_{N 1} \theta_{N 1}(k) & -a_{N 2} \theta_{N 2}(k) & \ldots & \sum_{j \in N_{i}} a_{N j} \theta_{N j}(k)
\end{array}\right] .
$$

and

$$
\tilde{\mathbf{L}}=\left[\begin{array}{cccc}
\sum_{j \in N_{i}} a_{1 j} & -a_{12} & \cdots & -a_{1 N} \\
-a_{21} & \sum_{j \in N_{i}} a_{2 j} & \cdots & -a_{2 N} \\
\vdots & \ddots & \ddots & \vdots \\
-a_{N 1} & -a_{N 2} & \cdots & \sum_{j \in N_{i}} a_{N j}
\end{array}\right] .
$$

Now, due to presence of random packet drop, expectation values of $\left(\mathbf{L}_{1}, \mathbf{L}_{2}\right)$ are, $E\left(\mathbf{L}_{1}\right)=\tilde{\mathbf{L}}(1-r)$ and $E\left(\mathbf{L}_{2}\right)=\tilde{\mathbf{L}} r$. And expectation value of (17) yields, 


$$
\begin{gathered}
E(\mathbf{e}(k+1))=\left(\mathbf{I}_{N} \otimes \mathbf{A}_{d}\right) E(\mathbf{e}(k))+\left(\tilde{\mathbf{L}}(1-r) \otimes \mathbf{B}_{d} \mathbf{K}\right) E(\mathbf{e}(k)) \\
+\left(\tilde{\mathbf{L}} r \otimes \mathbf{B}_{d} \mathbf{K}\right) E(\mathbf{e}(k-1))+\mathbf{B}_{d} E(\mathbf{w}(k)) .
\end{gathered}
$$

Now, using the system (21) the proof of Theorem 1 is described in the following.

\section{B. Proof of Theorem 1}

Defining the Lyapunov function candidate as:

$$
V(k)=\mathbf{e}^{T}(k) \mathbf{P e}(k)+\mathbf{e}^{T}(k-1) \mathbf{Q e}(k-1)
$$

for the inequality,

$$
V(k+1)-V(k)+\mathbf{y}_{e}^{T}(k) \mathbf{y}_{e}(k)-\gamma^{2} \mathbf{w}^{T}(k) \mathbf{w}(k)<\mathbf{0} .
$$

obtained from bounded real lemma (BRL) [21] which satisfies bounded $H_{\infty}$ norm $\gamma$.

However, since, $E\left(\mathbf{L}_{1}\right)=\tilde{\mathbf{L}}(1-r)$ and $E\left(\mathbf{L}_{2}\right)=\tilde{\mathbf{L}} r$, therefore, taking expectation value of (23), the following holds [21]:

$$
E(\Delta V(k))+E\left(\mathbf{y}_{e}^{T}(k) \mathbf{y}_{e}(k)-\gamma^{2} \mathbf{w}^{T}(k) \mathbf{w}(k)\right)<\mathbf{0} .
$$

From (24) as in [11],

$$
\begin{aligned}
E(\Delta V(k))=E(V(k+1)-V(k)) & \\
= & \mathbf{e}^{T}(k+1) \mathbf{P e}(k+1)+\mathbf{e}^{T}(k) \mathbf{Q e}(k) . \\
& -\mathbf{e}^{T}(k) \mathbf{P e}(k)+\mathbf{e}^{T}(k-1) \mathbf{Q e}(k-1)
\end{aligned} .
$$

Using(17) in (25) yields,

$$
\begin{aligned}
& {\left[\begin{array}{lll}
\mathbf{e}^{T}(k) & \mathbf{e}^{T}(k-1) & \mathbf{w}^{T}(k)
\end{array}\right] \times} \\
& {\left[\begin{array}{l}
\left(\left(\mathbf{I}_{N} \otimes \mathbf{A}_{d}\right)+\left(\tilde{\mathbf{L}}(1-r) \otimes \mathbf{B}_{d} \mathbf{K}\right)\right)^{T} \mathbf{P} \\
\times\left(\left(\mathbf{I}_{N} \otimes \mathbf{A}_{d}\right)+\left(\tilde{\mathbf{L}}(1-r) \otimes \mathbf{B}_{d} \mathbf{K}\right)\right) \\
+\mathbf{Q}-\mathbf{P}
\end{array}\right)\left(\left(\begin{array}{l}
\left(\mathbf{I}_{N} \otimes \mathbf{A}_{d}\right)+ \\
\left(\tilde{\mathbf{L}}(1-r) \otimes \mathbf{B}_{d} \mathbf{K}\right)
\end{array}\right)^{T}\right)} \\
& * \quad\left(\begin{array}{l}
\left(\tilde{\mathbf{L}} r \otimes \mathbf{B}_{d} \mathbf{K}\right)^{T} \mathbf{P} \times \\
\left(\tilde{\mathbf{L}} r \otimes \mathbf{B}_{d} \mathbf{K}\right)-\mathbf{Q}
\end{array}\right) \\
& \left.\begin{array}{c}
\left(\left(\mathbf{I}_{N} \otimes \mathbf{A}_{d}\right)+\left(\tilde{\mathbf{L}}(1-r) \otimes \mathbf{B}_{d} \mathbf{K}\right)\right)^{T} \mathbf{P}\left(\mathbf{I}_{N} \otimes \mathbf{B}_{d}\right) \\
\left(\tilde{\mathbf{L}} r \otimes \mathbf{B}_{d} \mathbf{K}\right)^{T} \mathbf{P}\left(\mathbf{I}_{N} \otimes \mathbf{B}_{d}\right) \\
\left(\mathbf{I}_{N} \otimes \mathbf{B}_{d}\right)^{T} \mathbf{P}\left(\mathbf{I}_{N} \otimes \mathbf{B}_{d}\right)
\end{array}\right]\left[\begin{array}{c}
\mathbf{e}(k) \\
\mathbf{e}(k-1) \\
\mathbf{w}(k)
\end{array}\right]<\mathbf{0}
\end{aligned}
$$

Again, as in [21],

$$
\begin{aligned}
& E\left(\mathbf{y}_{e}^{T}(k) \mathbf{y}_{e}(k)-\gamma^{2} \mathbf{w}^{T}(k) \mathbf{w}(k)\right) \\
& =\left[\begin{array}{lll}
\mathbf{e}^{T}(k) & \mathbf{e}^{T}(k-1) & \mathbf{w}^{T}(k)
\end{array}\right] \times \\
& {\left[\begin{array}{ccc}
\left(\mathbf{I}_{N} \otimes \mathbf{C}\right)^{T}\left(\mathbf{I}_{N} \otimes \mathbf{C}\right) & \mathbf{0} & \mathbf{0} \\
\mathbf{0} & \mathbf{0} & \mathbf{0} \\
\mathbf{0} & \mathbf{0} & -\gamma^{2} \otimes \mathbf{I}_{N}
\end{array}\right]\left[\begin{array}{c}
\mathbf{e}(k) \\
\mathbf{e}(k-1) \\
\mathbf{w}(k)
\end{array}\right]}
\end{aligned}
$$

By adding (26) and (27) and for non-zero $\mathbf{z}^{T}=\left[\begin{array}{lll}\mathbf{e}^{T}(k) & \mathbf{e}^{T}(k-1) & \mathbf{w}^{T}(k)\end{array}\right]$ yields:

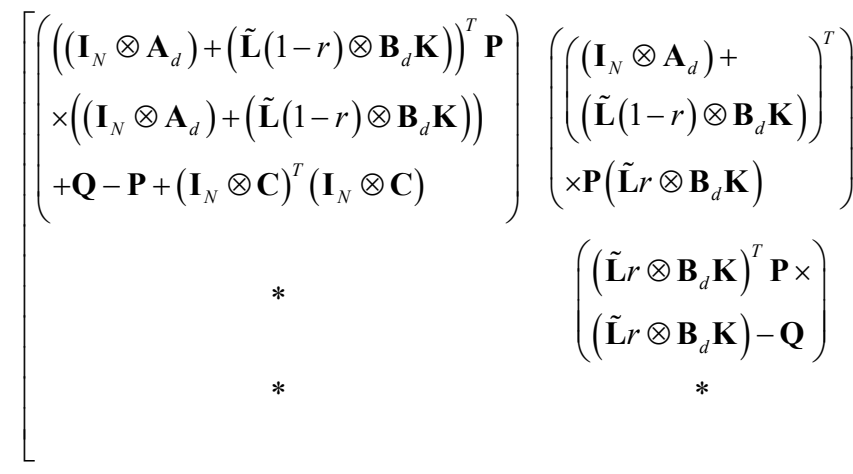

$$
\left.\begin{array}{c}
\left(\left(\mathbf{I}_{N} \otimes \mathbf{A}_{d}\right)+\left(\tilde{\mathbf{L}}(1-r) \otimes \mathbf{B}_{d} \mathbf{K}\right)\right)^{T} \mathbf{P}\left(\mathbf{I}_{N} \otimes \mathbf{B}_{d}\right) \\
\left(\tilde{\mathbf{L}} r \otimes \mathbf{B}_{d} \mathbf{K}\right)^{T} \mathbf{P}\left(\mathbf{I}_{N} \otimes \mathbf{B}_{d}\right) \\
\left(\mathbf{I}_{N} \otimes \mathbf{B}_{d}\right)^{T} \mathbf{P}\left(\mathbf{I}_{N} \otimes \mathbf{B}_{d}\right)-\gamma^{2} \otimes \mathbf{I}_{N}
\end{array}\right]<\mathbf{0} \text {. (28) }
$$

Now (28) can be re-written as:

$\left[\begin{array}{ccc}\mathbf{Q}-\mathbf{P} & \mathbf{0} & \mathbf{0} \\ \mathbf{0} & -\mathbf{Q} & 0 \\ \mathbf{0} & \mathbf{0} & -\gamma^{2} \otimes \mathbf{I}_{N}\end{array}\right]+\left[\begin{array}{c}\left(\mathbf{I}_{N} \otimes \mathbf{C}\right)^{T} \\ \mathbf{0} \\ \mathbf{0}\end{array}\right]\left[\left(\mathbf{I}_{N} \otimes \mathbf{C}\right) \quad \mathbf{0} \quad \mathbf{0}\right]$

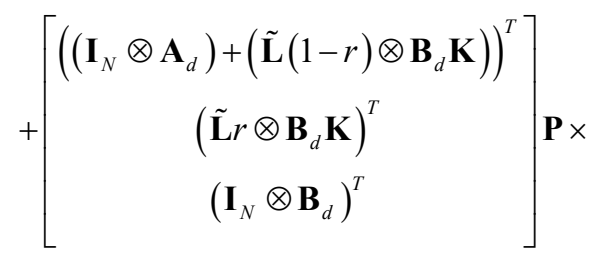

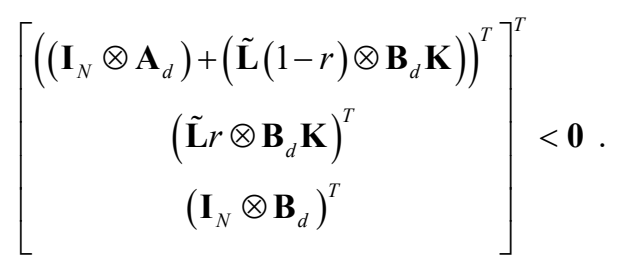

Using Schur complement [22] of (29) yields,

$$
\left[\begin{array}{cccc}
\mathbf{Q}-\mathbf{P} & & * & * \\
\mathbf{0} & & -\mathbf{Q} & * \\
\mathbf{0} & & \mathbf{0} & -\gamma^{2} \otimes \mathbf{I}_{N} \\
\left(\left(\mathbf{I}_{N} \otimes \mathbf{A}_{d}\right)+\tilde{\mathbf{L}}(1-r) \otimes \mathbf{B}_{d} \mathbf{K}\right) & \tilde{\mathbf{L}} r \otimes \mathbf{B}_{d} \mathbf{K} & \mathbf{I}_{N} \otimes \mathbf{B}_{d} \\
\mathbf{I}_{N} \otimes \mathbf{C} & & \mathbf{0} & \mathbf{0} \\
& * & * \\
& * & * \\
& * & * \\
& -\mathbf{P}^{-1} & * \\
& \mathbf{0} & -\mathbf{I}_{N}
\end{array}\right]<\mathbf{0}
$$

Now multiplying by $\operatorname{diag}\left\{\mathbf{P}^{-1}, \mathbf{P}^{-1}, \mathbf{I}_{N}, \mathbf{I}_{N}, \mathbf{I}_{N}\right\}$ in both side of (30) and defining $\mathbf{P}^{-1}=\mathbf{I}_{N} \otimes \mathbf{P}_{0}^{-1}$ and $\mathbf{K}=\mathbf{Y} \mathbf{P}_{0}$ yields:

$\left[\begin{array}{ccc}\mathbf{P}^{-1} \mathbf{Q} \mathbf{P}^{-1}-\mathbf{P}^{-1} & * & * \\ \mathbf{0} & -\mathbf{P}^{-1} \mathbf{Q} \mathbf{P}^{-1} & * \\ \mathbf{0} & \mathbf{0} & -\gamma^{2} \otimes \mathbf{I}_{N} \\ \left(\left(\mathbf{I}_{N} \otimes \mathbf{A}_{d}\right) \mathbf{P}^{-1}+\tilde{\mathbf{L}}(1-r) \otimes \mathbf{B}_{d} \mathbf{Y}\right) & \tilde{\mathbf{L}} r \otimes \mathbf{B}_{d} \mathbf{Y} & \mathbf{I}_{N} \otimes \mathbf{B}_{d} \\ \left(\mathbf{I}_{N} \otimes \mathbf{C}\right) \mathbf{P}^{-1} & \mathbf{0} & \mathbf{0}\end{array}\right.$




$$
\left.\begin{array}{cc}
* & * \\
* & * \\
* & * \\
-\mathbf{P}^{-1} & * \\
\mathbf{0} & -\mathbf{I}_{N}
\end{array}\right]<\mathbf{0}
$$

It is seen that (31) is a nonconvex LMI due to presence of constraints $\left(\mathbf{P}^{-1}, \mathbf{Q}^{-1}\right)$. This nonconvexity problem of (31) can be overcome by assuming

$$
\mathbf{P}^{-1} \mathbf{Q} \mathbf{P}^{-1}=\mathbf{M} \text { and } \mathbf{P}^{-1}=\overline{\mathbf{P}},
$$

where $\mathbf{Q}=\mathbf{I}_{N} \otimes \mathbf{Q}_{0}$.

Thus, using (32), (31) takes the LMI form as:

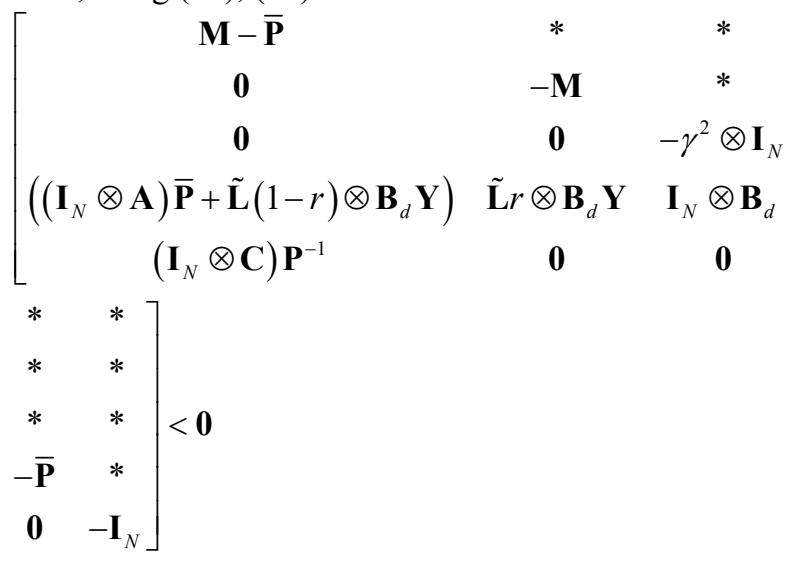

and (32) yields the LMI form

$$
\left[\begin{array}{cc}
-\mathbf{M} & \overline{\mathbf{P}} \\
\overline{\mathbf{P}} & -\overline{\mathbf{Q}}
\end{array}\right] \leq \mathbf{0}
$$

where $\overline{\mathbf{Q}}=\mathbf{Q}^{-1}$.

Now, (33) can be re-written as:

$\left[\begin{array}{cccc}-\overline{\mathbf{P}} & * & * & * \\ \mathbf{0} & -\mathbf{M} & * & * \\ \mathbf{0} & \mathbf{0} & -\gamma^{2} \otimes \mathbf{I}_{N} & * \\ \left(\left(\mathbf{I}_{N} \otimes \mathbf{A}\right) \overline{\mathbf{P}}+\tilde{\mathbf{L}}(1-r) \otimes \mathbf{B}_{d} \mathbf{Y}\right) & \tilde{\mathbf{L}} r \otimes \mathbf{B}_{d} \mathbf{Y} & \mathbf{I}_{N} \otimes \mathbf{B}_{d} & -\overline{\mathbf{P}}\end{array}\right]$

$+\left[\begin{array}{c}\overline{\mathbf{P}}^{T} \\ \mathbf{0} \\ \mathbf{0} \\ \mathbf{0}\end{array}\right] \mathbf{Q}\left[\begin{array}{llll}\overline{\mathbf{P}} & \mathbf{0} & \mathbf{0} & \mathbf{0}\end{array}\right]+$

$\left[\begin{array}{c}\overline{\mathbf{P}}^{T}\left(\mathbf{I}_{N} \otimes \mathbf{C}\right)^{T} \\ \mathbf{0} \\ \mathbf{0}\end{array}\right]\left[\left(\mathbf{I}_{N} \otimes \mathbf{C}\right) \overline{\mathbf{P}} \quad \mathbf{0} \quad \mathbf{0} \quad \mathbf{0}\right]<\mathbf{0}$.

Using Schur complement of (35), (32) and $\overline{\mathbf{P}}=\mathbf{P}^{-1}, \overline{\mathbf{Q}}=\mathbf{Q}^{-1}$, the LMI can be obtained the form (13).

\section{NUMERICAL RESULTS}

This section represents the results to demonstrate the effectiveness of the proposed methodology for achieving cooperative motion of platoon considering seven different network topologies i.e. PF, PLF, BPF [4], BPLF, TPF [5], TBPF [4] and all-to-all [6] with different number of homogeneous follower vehicles in discrete time domain with specified sampling time $T_{\mathrm{s}}=0.1 \mathrm{~s}$. A constant spacing policy of $25 \mathrm{~m} \mathrm{[4]} \mathrm{is} \mathrm{used} \mathrm{among} \mathrm{the} \mathrm{vehicles} \mathrm{in} \mathrm{a} \mathrm{platoon.} \mathrm{Again,}$ for all the homogeneous followers it is assumed that there is no collision at initial time $\mathrm{t}=0 \mathrm{~s}$ and the initial states are random in nature. The time lag is chosen as $\tau=0.4 s$ [4], [6]. The Leader's speed is considered constant at $72 \mathrm{~km} / \mathrm{hr}$ [23]. In this paper, the external disturbance input to the acceleration of all followers has been considered as a square wave of amplitude 1, starting at $t=120 \mathrm{~s}$ and thereafter continued for the period of $20 s$ as shown in Figure 1, with limited energy which means $\left\|w_{i}(k)\right\|_{L_{2}}$ is bounded. Under such scenario, the $\gamma$ measures the robustness of a platoon which is defined by $\gamma \geq \sup \left(\left\|\mathbf{y}_{e}(k)\right\|_{L_{2}} /\|\mathbf{w}(k)\|_{L_{2}}\right)$. Therefore, $\gamma$ describes the sensitivity or attenuation effect of the energy of external disturbances.

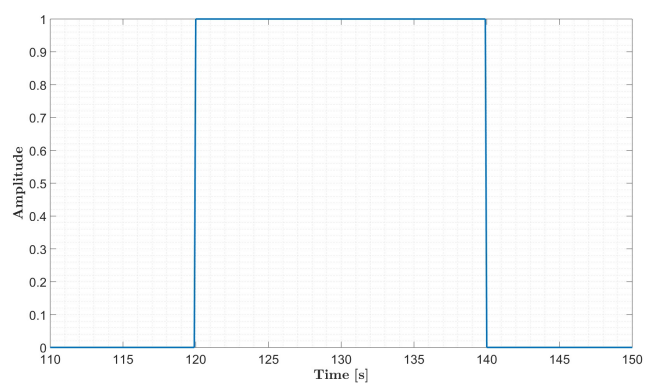

Figure 1: Disturbance input

Now, the variation of $\gamma^{2}$ has been depicted in Figure 2 to demonstrate the effect of network topologies, platoon members and packet drop in platoon by solving the designed LMI (13). The LMI has been solved using YALMIP toolbox [24] and SeDuMi solver [25] considering seven different network topologies [5,9], different number of platoon members $(N=3$ to 10$)$ and under varying packet drop rate $(r$ $=0.1$ to 0.5 representing $10 \%$ to $50 \%$ ) (colour bar). It can be observed form the Figure 2 (a) and (b) that under 20\% packet drop, the value of $\gamma^{2}$ is increasing when the number of platoon members $(N)$ are higher and this is same for all the seven different network topologies i.e. PF, PLF,BPF, BPLF, TPF, TBPF, all-to all. In Figure 2 (a) and (b), the value of $\gamma^{2}$ is higher when the amount of information exchange between platoon members is less. Also, it is evident that the magnitude of $\gamma^{2}$ is more for the topologies where the leader is connected only with the first follower in a platoon compared to all-to-all network topology under $20 \%$ packet drop. Next, Figure 2 (b) compares the variation of $\gamma^{2}$ for $N=$ 3 to 10 under $20 \%$ packet drop scenario for four different network topologies i.e. PF with PLF (left) and BPF with BPLF (right). It is seen form the Figure 2 (b) the value of $\gamma^{2}$ is increasing with the platoon number $(N)$ and this value is higher when leader is connected only with first follower i.e. PF and BPF compared to all leader following i.e. PLF and BPLF under $20 \%$ packet drop as expected. In Figure 2 (c), all-to-all network topology has been considered to depict the variation of $\gamma^{2}$ for different platoon $(N)$ i.e. 3 to 10 and different packet drop rate $(r)$ i.e. $10 \%$ to $50 \%$ (colour bar). It is seen from the Figure 2 (c) that the value of $\gamma^{2}$ is becoming larger when number of vehicles in a platoon and rate of packet drop is increasing. It can be noted that with the variation of the rate of packet drop, the value of $\gamma^{2}$ is not varying a large amount because the proposed algorithm is 
designed and validated under the consideration of single packet drop.

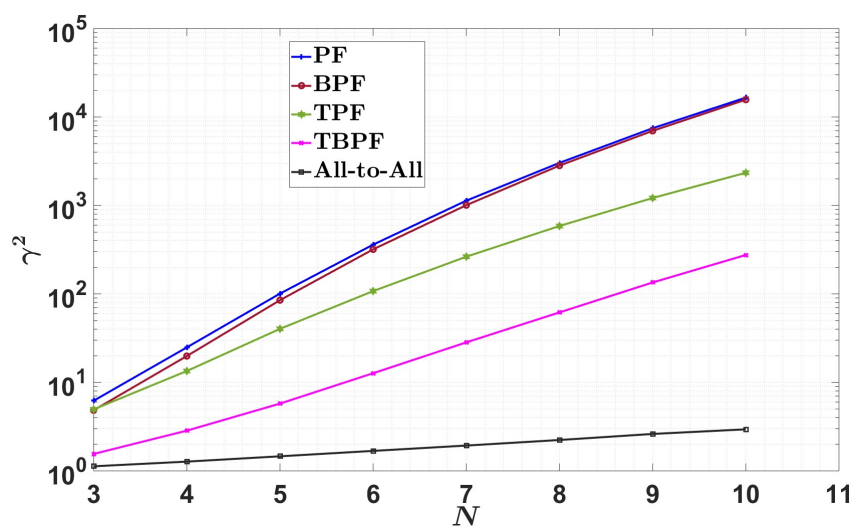

(a)
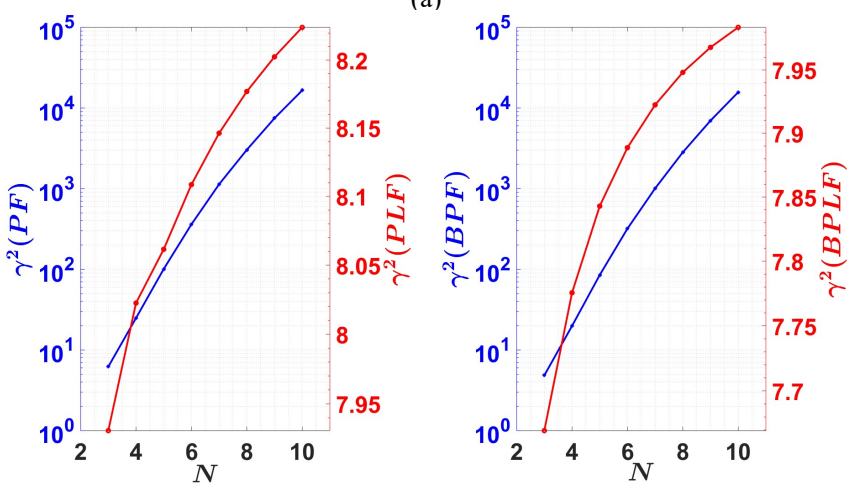

(b)

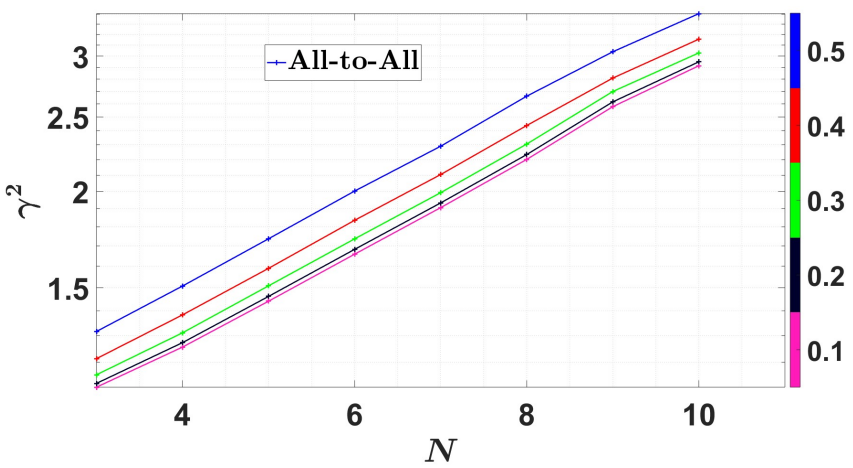

(c)

Figure 2: Variation of $\gamma^{2}$ for different network topologies with different number of follower vehicles under different packet drop rate (a) effect of network topology under $20 \%$ packet drop (b) effect of leader information under $20 \%$ packet drop (c) effect of packet drop (i.e. $10 \%$ to $50 \%$ ).

Next, Figure 3-5 shows the effectiveness of the designed controller to achieve control objectives (7) and that has been demonstrated in terms of time responses considering 10 followers in a platoon for two different network topologies i.e. BPF and BPLF under 20\% packet drop [7]. By solving the LMI (13), the controller gains $\mathbf{K}=[-0.5528,-6.5034$, $2.5130]$ and $\mathbf{K}=[-3.0506,-3.9947,-1.5223]$ are obtained for BPF and BPLF topology, respectively. It is assumed that the follower states are mismatched at time instant $t=0 \mathrm{~s}$ e.g., upto $2 \mathrm{~m}$ inter vehicular distance error $\left(e_{i-1, i}\right)$ among the consecutive platoon members and hence the variation in velocities $\left(v_{\mathrm{i}}\right)$ of followers. It is seen from Figure 3-4 that all vehicle dynamics are converging to desired behaviours i.e. zero inter-vehicular distance error, matching to leader's velocity, zero accelerations $\left(a_{\mathrm{i}}\right)$ when the controllers are activated. It is also seen that the converging time i.e. $40 \mathrm{~s}$ of the follower states in the BPF topology is higher than the converging time $(3.5 \mathrm{~s})$ of states in the BPLF topology under $20 \%$ packet drop since leader vehicle is connected to all the followers in BPLF topology as in [5]. Figure 4 depicts the behaviour of follower's states when the external disturbances shown in Figure 1 is applied to each follower under $20 \%$ packet drop. Under such scenario, the inter-vehicular distance error (maximum $15.3 \mathrm{~m}$ ) and settling time is higher when BPF topology is used compared to BPLF topology where maximum inter-vehicular distance error is $0.35 \mathrm{~m}$. For BPLF topology the inter-vehicular distance error exist only for the first follower and other followers are maintaining almost zero error. This is because all the followers have zero initial error (at steady state) and they are also pinned to the leader, thereby maintaining same dynamic evaluation [5]. Again, the amplification of velocity is also higher for BPF topology compared to BPLF topology. It is also noted that each vehicle has maintained almost same velocity and acceleration when BPLF topology is considered.
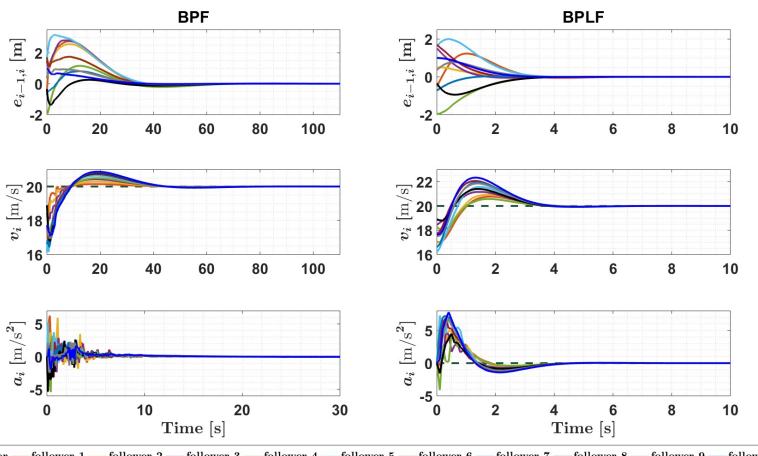

Figure 3: Steady state response for 10 follower vehicles in a platoon under $20 \%$ packet drop rate with BPF (left panel) and BPLF topology (right panel).
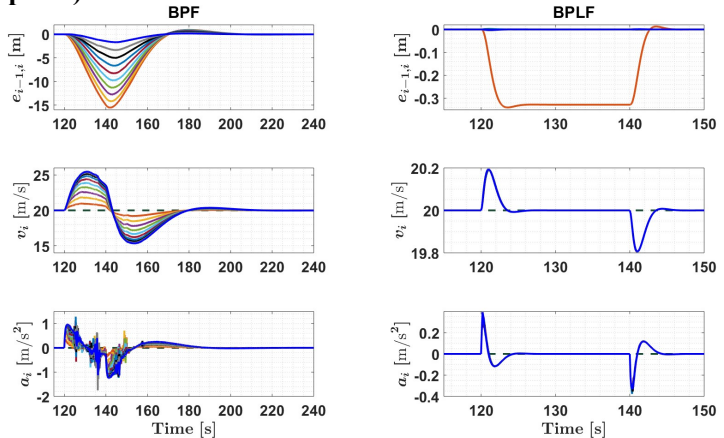

Figure 4: Time response of the follower's states under external disturbance and $20 \%$ packet drop for BPF (left panel) and BPLF topology (right panel).
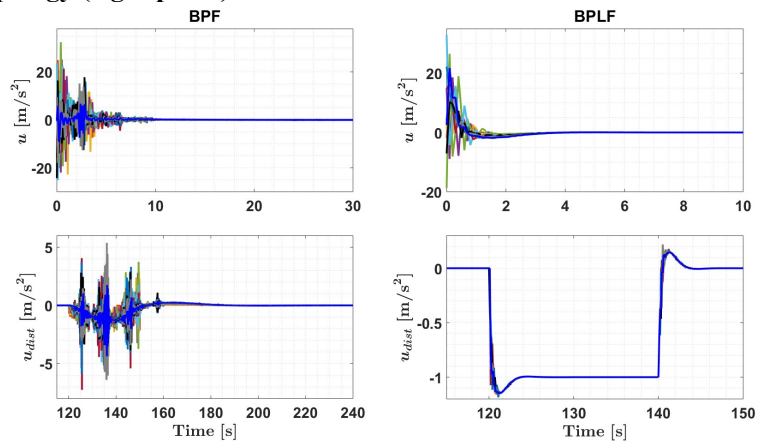

- follower $1-$ follower $2-$ follower $3-$ follower $4-$ follower $5-$ follower 6 -follower 7 - follower 8 - follower $9-$ follower 10
Figure 5: Control inputs to the followers at steady state (upper panel) and external disturbances (lower panel) under $20 \%$ packet drop. 
From Figure 5, it is seen that all the control inputs are converging to zero for both topologies before and after acting of external disturbances to each follower. Also, highly oscillatory nature of control inputs for the BPF topology describes the requirement of high control effort compared to the BPLF topology.

\section{CONCLUSION}

In this paper, an LMI based distributed state feedback controller satisfying bounded $H_{\infty}$ norm has been designed for vehicle platoon under single packet drop. The variation of bounded $H_{\infty}$ norm for different network topologies and number of platoon members under packet drop, has also been presented. The designed controller has been tested for 10 follower vehicles in a platoon considering two different network topologies under $20 \%$ packet drop, as well. The simulation results demonstrated the effectiveness of the proposed controller for maintaining desired performances for vehicle platooning under random packet drop and external disturbances. As a scope of future work, the proposed method can be extended under the scenario of multiple packet drop.

\section{Acknowledgment}

This work is supported by Jaguar Land Rover and the UK-EPSRC grant EP/N01300X/1 as part of the jointly funded Towards Autonomy: Smart and Connected Control (TASCC).

\section{REFERENCES}

[1] J. Karl Hedrick, Masayoshi Tomizuka, and P. Varaiya. "Control issues in automated highway systems", IEEE Control Systems Magazine 14, no. 6 (1994): 21-32

[2] Shengbo Eben Li, Yang Zheng, Keqiang Li, and Jianqiang Wang, "An overview of vehicular platoon control under the four-component framework", In 2015 IEEE Intelligent Vehicles Symposium (IV), pp. 286-291. IEEE, 2015.

[3] Dongyao Jia, and Dong Ngoduy, "Enhanced cooperative carfollowing traffic model with the combination of V2V and V2I communication", Transportation Research Part B: Methodological 90 (2016): 172-191.

[4] Shengbo Eben Li, Xiaohui Qin, Yang Zheng, Jianqiang Wang, Keqiang Li, and Hongwei Zhang, "Distributed platoon control under topologies with complex eigenvalues: stability analysis and controller synthesis" IEEE Transactions on Control Systems Technology 99 (2017): 1-15.

[5] Yang Zheng, Shengbo Eben Li, Jianqiang Wang, Dongpu Cao, and Keqiang Li, "Stability and scalability of homogeneous vehicular platoon: Study on the influence of information flow topologies", IEEE Transactions on intelligent transportation systems 17, no. 1 (2015): 14-26.

[6] Umberto Montanaro, Saber Fallah, Mehrdad Dianati, David Oxtoby, Tom Mizutani, and Alexandros Mouzakitis, "On a Fully SelfOrganizing Vehicle Platooning Supported by Cloud Computing" In 2018 Fifth International Conference on Internet of Things: Systems, Management and Security, pp. 295-302. IEEE, 2018.

[7] Ye Tang, Maode Yan, Panpan Yang, and Lei Zuo, "Consensus based control algorithm for vehicle platoon with packet losses" In 2018 37th Chinese Control Conference (CCC), pp. 7684-7689. IEEE, 2018.

[8] Dunbar, William B., and Derek S. Caveney, "Distributed receding horizon control of vehicle platoons: Stability and string stability" IEEE Transactions on Automatic Control 57, no. 3 (2011): 620-633.

[9] Zheng, Yang, Shengbo Eben Li, Keqiang Li, Francesco Borrelli, and J. Karl Hedrick, "Distributed model predictive control for heterogeneous vehicle platoons under unidirectional topologies",
IEEE Transactions on Control Systems Technology 25, no. 3 (2016): 899-910.

[10] Yang Zheng, Shengbo Eben Li, Keqiang Li, and Wei Ren, "Platooning of connected vehicles with undirected topologies: Robustness analysis and distributed H-infinity controller synthesis", IEEE Transactions on Intelligent Transportation Systems 19, no. 5 (2017): 1353-1364.

[11] Ya-Jun Pan, Herbert Werner, Zipeng Huang, and Marcus Bartels. "Distributed cooperative control of leader-follower multi-agent systems under packet dropouts for quadcopters", Systems \& Control Letters 106 (2017): 47-57.

[12] J. W. Kwon and D. Chwa, Adaptive bidirectional platoon control using a coupled sliding mode control method. IEEE Transactions on Intelligent Transportation Systems, 15(5), pp.2040-2048, 2014.

[13] L. Xu, W. Zhuang, G. Yin and C. Bian, "Stable Longitudinal Control of Heterogeneous Vehicular Platoon With Disturbances and Information Delays", IEEE Access, 6, pp.69794-69806, 2018.

[14] H. Zhou, R. Saigal, F. Dion and L. Yang, "Vehicle platoon control in high-latency wireless communications environment: Model predictive control method", Transportation Research Record, 2324(1), pp.81-90, 2012.

[15] C. Lei, E.M. Van Eenennaam, W.K. Wolterink, G. Karagiannis, G. Heijenk and J. Ploeg, "Impact of packet loss on CACC string stability performance", In 2011 11th International Conference on ITS Telecommunications, pp. 381-386, August, 2011, IEEE.

[16] S. Wen and G. Guo, "Cooperative control and communication of connected vehicles considering packet dropping rate", International Journal of Systems Science, 49(13), pp.2808-2825, 2018.

[17] Ge Guo, and Wei Yue. "Hierarchical platoon control with heterogeneous information feedback", IET control theory \& applications 5, no. 15 (2011): 1766-1781.

[18] S. Öncü, J. Ploeg, N. Van de Wouw and H. Nijmeijer, "Cooperative adaptive cruise control: Network-aware analysis of string stability", IEEE Transactions on Intelligent Transportation Systems, 15(4), pp.1527-1537, 2014.

[19] Liwei Xu, Weichao Zhuang, Guodong Yin,Chentong Bian, and Huawei Wu, "Modeling and Robust Control of HeterogeneousVehicle Platoons on Curved Roads Subject to Disturbances and Delays", IEEE Transactions on Vehicular Technology, DOI 10.1109/TVT.2019.2941396,2019.

[20] A. Pawar and Y.J. Pan, "Leader-following consensus control of multiagent systems with communication delays \& random packet loss" In 2016 American Control Conference (ACC) (pp. 4464-4469), July, 2016, IEEE.

[21] Zidong Wang, Fuwen Yang, Daniel WC Ho, and Xiaohui Liu. "Robust Control for Networked Systems With Random Packet Losses", IEEE Transactions on Systems, Man, and Cybernetics, Part B (Cybernetics) 37, no. 4 (2007): 916-924.

[22] David Carlson, "What are Schur complements, anyway?" Linear Algebra and its Applications 74 (1986): 257-275.

[23] Jeroen C. Zegers, Elham Semsar-Kazerooni, Jeroen Ploeg, Nathan van de Wouw, and Henk Nijmeijer, "Consensus control for vehicular platooning with velocity constraints", IEEE Transactions on Control Systems Technology 26, no. 5 (2018): 1592-1605.

[24] https://yalmip.github.io/

[25] Jos F. Sturm, "Using SeDuMi 1.02, a MATLAB toolbox for optimization over symmetric cones" Optimization methods and software 11, no. 1-4 (1999): 625-653. 